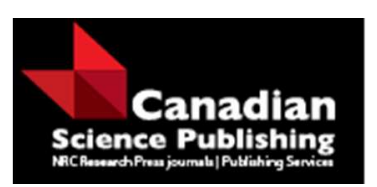

Canadian Journal of Physics

Revue canadienne de physique

\title{
Bianchi type-V modified holographic Ricci dark energy models in Saez-Ballester theory of gravitation
}

\begin{tabular}{|r|l|}
\hline Journal: & Canadian Journal of Physics \\
\hline Manuscript ID & cjp-2016-0464.R1 \\
\hline Manuscript Type: & Article \\
\hline Date Submitted by the Author: & $19-$ Oct-2016 \\
\hline Complete List of Authors: & REDDY, DRK; Andhra University, Waltair, Mathematics \\
\hline Keyword: & $\begin{array}{l}\text { Hybrid expansion law,, Saz-Ballester theory, Bianchi type-V metric, } \\
\text { deceleration parameter, holographic dark energy }\end{array}$ \\
\hline
\end{tabular}

SCHOLARONE $^{m}$
Manuscripts 


\title{
Bianchi type-V modified holographic Ricci dark energy models in Saez-Ballester theory of gravitation
}

\author{
D.R.K.Reddy* \\ *Department of Applied Mathematics, Andhra University, Visakhapatnam, India-530003 \\ *reddy_einstein@yahoo.com
}

\begin{abstract}
We have investigated Bianchi type-V universe filled with matter and modified holographic Ricci dark energy in a scalar-tensor theory proposed by SaezBallester (Phys. Lett. A., 113, 467, 1986). To get a determinate solution, we have used (i) hybrid expansion law (Akarsu et al. JCAP, 01, 022, 2014), (ii) varying deceleration parameter (Mishra et al. Int. J. Theor. Phys. 52, 2546, 2013) and (iii) linearly varying deceleration parameter (Akarsu and Dereli, Int. J. Theor. Phys. 51, 612, 2012). The various physical and geometrical aspects of the models are also discussed.
\end{abstract}

Key words: Bianchi type-V metric, Saez-Ballester theory, Hybrid expansion law, varying deceleration parameter, holographic dark energy.

\section{Introduction}

The present day universe is in a state of accelerated expansion which has been confirmed by the recent cosmological observations $[1,2]$. The data also suggests that the universe is dominated by two dark components containing dark matter and dark energy. Dark matter, a matter without pressure, is mainly used to explain galactic curves and structure formation of the universe while dark energy, an exotic energy with negative pressure, is used to explain the present cosmic accelerated expansions. The study of dark energy models in some significant modified theories of gravitation is one of the approaches to explain early inflation and late time acceleration of the universe.

In recent years, holographic dark energy (HDE) models have received considerable attention to describe dark energy cosmological models. According to the holographic principle, the number of degrees of freedom in a bounded system should be finite and is related to the area of its boundary [3]. It is argued that this model may solve the 
cosmological constant problem and some other issues. Several aspects of holographic dark energy have been investigated by Cohen et al. [4] and Hsu [5], Gao et al. [6] have proposed a HDE model, where the future event horizon is replaced by the inverse of the Ricci scalar curvature, and thismodel is named as "Ricci dark energy model" (RDE), that is, a holographic Ricci dark energy model, whose length scale is the inverse of the Ricci curvature scalar, i.e. $L \approx|R|^{\frac{-1}{2}}$. Granda and Oliveros [7] suggested a new holographic Ricci dark energy model. Later, Chen and Jing [8] modified this model by assuming the density of dark energy contains the Hubble parameter $H$, the first order and the second order derivatives (i.e., $\dot{H}$ and $\ddot{H}$ ). The expression of the energy density of dark energy is given by

$$
\rho_{\Lambda}=3 \eta_{1} \ddot{H} H^{-1}+3 \eta_{2} \dot{H}+3 \eta_{3} H^{2}
$$

where $\eta_{1}, \eta_{2}$ and $\eta_{3}$ are three arbitrary dimensionless parameters. Recently, Sarkar [9], Adhav et al. [10], Kiran et al. [11,12], Umadevi and Ramesh [13] have investigated minimally interacting and interacting holographic dark energy Bianchi models in general relativity and in scalar-tensor theories of gravitation. Very recently, Kiran et al. [14] and Reddy et al. [15] have discussed Bianchi type minimally interacting holographic dark energy models using linearly varying deceleration parameter proposed by Akarsu and Dereli [16]. Das and Sultana [17,18] have studied Bianchi type anisotropic modified holographic Ricci dark energy cosmological models. Rahman and Ansari [19] have investigated interacting holographic polytropic gas model of dark energy with hybrid expansion law in Bianchi type- $\mathrm{VI}_{0}$ space time while Rahman and Ansari [20] have studied interacting generalized ghost polytropic gas model of dark energy with a specific Hubble parameter in LRS Bianchi type-II space time.

Inspired by above investigations in this paper, we investigate anisotropic Bianchi type- $\mathrm{V}$ models in the presence of anisotropic modified holographic Ricci dark energy. The plan of this paper is the following: In section 2, we obtain the field equations of Saez-Ballester theory in the presence of matter and modified holographic Ricci dark 
energy in Bianchi type- $\mathrm{V}$ space time. By solving the field equations using various average scale factors, we present the modified holographic Ricci dark energy model in section 3. In section 4, we compute the physical and kinematical parameters of the model and discuss their physical significance. The last section contains some concluding remarks.

\section{Metric and field equations}

We consider a spatially homogeneous Bianchi type- $\mathrm{V}$ metrics of the form

$$
d s^{2}=d t^{2}-A^{2} d x^{2}-B^{2} e^{-2 x} d y^{2}-C^{2} e^{-2 x} d z^{2}
$$

where $A, B, C$ are functions of cosmic time $t$.

Saez-Ballester [21] field equations for combined scalar and tensor fields in the presence of dark matter and holographic dark energy are given by

$$
R_{i j}-\frac{1}{2} g_{i j} R-w \phi^{n}\left(\varphi_{, i} \varphi_{, j}-\frac{1}{2} g_{i j} \varphi_{, k} \varphi^{, k}\right)=-\left(T_{i j}+\overline{T_{i j}}\right)
$$

where $R_{i j}$ is the Ricci tensor, $R$ is the Ricci scalar, $w$ and $\mathrm{n}$ arbitrary dimensionless constants and $8 \pi G=c=1$ in the relativistic units.

The energy momentum tensor for matter and the dark energy are defined as

$$
\left.\begin{array}{l}
T_{i j}=\rho_{M} u_{i} u_{j} \\
\overline{T_{i j}}=\left(\rho_{\lambda}+p_{\lambda}\right) u_{i} u_{j}-g_{i j} p_{\lambda}
\end{array}\right\}
$$

The energy momentum tensor of dark energy can be parameterized as

$$
\bar{T}_{i j}=\operatorname{diag}\left[1,-\omega_{\lambda},-\left(\omega_{\lambda}+\delta_{y}\right),-\left(\omega_{\lambda}+\delta_{z}\right)\right] \rho_{\lambda}
$$

where $\omega_{\lambda}=\frac{p_{\lambda}}{\rho_{\lambda}}$ is the equation of state (EoS) parameter of dark energy and $\rho_{M}, \rho_{\lambda}$ are the energy densities of matter and the dark energy and $p_{\lambda}$ is the pressure of the dark energy.

Here skewness parameters $\delta_{y}$ and $\delta_{z}$ are the deviations from $\omega_{\lambda}$ along $y$ and $z$ directions respectively. 
The scalar field $\varphi$ satisfies the following equation

$$
2 \varphi^{n} \varphi_{; i}^{, i}+n \varphi^{n-1} \varphi_{, k} \varphi^{, k}=0
$$

Also, the energy conservation equation is

$$
T_{; j}^{i j}+\overline{T_{; j}^{i j}}=0
$$

In a commoving coordinate system, Saez-Ballester field equations (3) for the metric (2) with the help of Eq. (4) can be, explicitly, written as

$$
\begin{aligned}
& \frac{\ddot{B}}{B}+\frac{\ddot{C}}{C}+\frac{\dot{B} \dot{C}}{B C}-\frac{1}{A^{2}}-\frac{1}{2} w \varphi^{n} \dot{\varphi}^{2}=-\omega_{\lambda} \rho_{\lambda}, \\
& \frac{\ddot{A}}{A}+\frac{\ddot{C}}{C}+\frac{\dot{A} \dot{C}}{A C}-\frac{1}{A^{2}}-\frac{1}{2} w \varphi^{n} \dot{\varphi}^{2}=-\left(\omega_{\lambda}+\delta_{y}\right) \rho_{\lambda}, \\
& \frac{\ddot{A}}{A}+\frac{\ddot{B}}{B}+\frac{\dot{A} \dot{B}}{A B}-\frac{1}{A^{2}}-\frac{1}{2} w \varphi^{n} \dot{\varphi}^{2}=-\left(\omega_{\lambda}+\delta_{z}\right) \rho_{\lambda}, \\
& \frac{\dot{A} \dot{B}}{A B}+\frac{\dot{B} \dot{C}}{B C}+\frac{\dot{C} \dot{A}}{C A}-\frac{1}{A^{2}}+\frac{1}{2} w \varphi^{n} \dot{\varphi}^{2}=\rho_{M}+\rho_{\lambda}, \\
& \frac{\dot{B}}{B}+\frac{\dot{C}}{C}-\frac{2 \dot{A}}{A}=0 . \\
& \ddot{\varphi}+\dot{\varphi}\left(\frac{\dot{A}}{A}+\frac{\dot{B}}{B}+\frac{\dot{C}}{C}\right)+\frac{n}{2} \frac{\dot{\varphi}^{2}}{\varphi}=0
\end{aligned}
$$

Also energy conservation equation (6) is given by

$$
\dot{\rho}_{\lambda}+\dot{\rho}_{M}+\left(\frac{\dot{A}}{A}+\frac{\dot{B}}{B}+\frac{\dot{C}}{C}\right)\left(\rho_{\lambda}+\rho_{M}+p_{\lambda}\right)+\left(\frac{\dot{B}}{B} \delta_{y}+\frac{\dot{C}}{C} \delta_{z}\right) \rho_{\lambda}=0
$$

Here over head denotes differentiation with respect to cosmic time $t$.

\section{Solutions and the models}

Integration of Eq. (11) yields

$$
A^{2}=k_{1} B C
$$


where $k_{1}$ is a constant of integration which can be chosen as unity without loss of generality, so that we have

$A^{2}=B C$

Now Eqs. (7)-(12) are a system of five independent equations in nine unknowns $A, B, C, \omega_{\lambda}, \rho_{\lambda}, \delta_{y}, \delta_{z}, \rho_{M}$ and $\varphi$. Hence to find a determinate solution we use the following physically plausible conditions:

(i) The shear scholar is proportional to scalar expansion so that we have [22]

$$
B=C^{k}
$$

(ii) The energy density of modified holographic Ricci dark energy given by Eq. (1) [8].

(iii) An ansatz for the average scale factor $a(t)$ of the universe.

Here we obtain three models choosing different ansatz for the average scale factor of the universe.

Model-1: Following Akarsu et al. [23], we consider an ansatz for the average scale factor as

$$
a(t)=(A B C)^{\frac{1}{3}}=a_{0} t^{\alpha_{1}} e^{\alpha_{2} t}
$$

where $\alpha_{1}$ and $\alpha_{2}$ are non-negative constants and $a_{0}$ is the present value of the scale factor. Eq. (17) is known as hybrid expansion law which is a combination of power law and an exponential function. It can be seen that $\alpha_{2}=0$ yields power law cosmology while $\alpha_{1}=0$ gives exponential law cosmology. Thus, the power-law and exponential law cosmologies are the special cases of hybrid expansion law cosmology. This choice of average scale factor gives a timedependent deceleration parameter. The solution gives inflation and radiation dominance era with subsequent transition from deceleration to acceleration. Recently, Shri Ram \& Chandel [24] and Santhi et al. [25] have studied Bianchi dark energy cosmological models with hybrid expansion law. Using equations (15), (16) and (17) we obtain the scale factors as 


$$
\begin{aligned}
& A=a_{0} t^{\alpha_{1}} e^{\alpha_{2} t} \\
& B=\left(a_{0} t^{\alpha_{1}} e^{\alpha_{2} t}\right)^{\frac{2 k}{k+1}} \\
& C=\left(a_{0} t^{\alpha_{1}} e^{\alpha_{2} t}\right)^{\frac{2}{k+1}}
\end{aligned}
$$

From Eqs. (12) and (18), we have the scalar field as

$$
\varphi^{\frac{n+2}{2}}=\frac{n+2}{2} \int\left(\varphi_{0} a_{0} t^{\alpha_{1}} e^{\alpha_{2} t}\right)^{-3} d t+\psi_{0}
$$

Using Eq. (18) in Eq. (2) we obtain the following model:

$$
d s^{2}=d t^{2}-\left(a_{0} t^{\alpha_{1}} e^{\alpha_{2} t}\right)^{2} d x^{2}-\left(a_{0} t^{\alpha_{1}} e^{\alpha_{2} t}\right)^{\frac{4 k}{k+1}} e^{-2 x} d y^{2}-\left(a_{0} t^{\alpha_{1}} e^{\alpha_{2} t}\right)^{\frac{4}{k+1}} e^{-2 x} d z^{2}
$$

\section{Physical discussion of the model}

Equation (25) describes the modified holographic Ricci dark energy model in Bianchi type-V space time in the frame work of Saez-Ballester scalar-tensor theory of gravitation with the following physical and kinematical parameters which are very important for physical discussion of the model.

Spatial volume is

$$
V=a^{3}=\left(a_{0} t^{\alpha_{1}} e^{\alpha_{2} t}\right)^{3}
$$

The average Hubble's parameter is

$$
H=\frac{\dot{a}}{a}=\frac{\alpha_{1}}{t}+\alpha_{2}
$$

The scalar expansion is

$$
\theta=3 H=3\left(\frac{\alpha_{1}}{t}+\alpha_{2}\right)
$$

The shear scalar is 


$$
\begin{aligned}
\sigma^{2} & =\frac{1}{3}\left(\left(\frac{\dot{A}}{A}\right)^{2}+\left(\frac{\dot{B}}{B}\right)^{2}+\left(\frac{\dot{C}}{C}\right)^{2}-\frac{\dot{A} \dot{B}}{A B}-\frac{\dot{C} \dot{B}}{C B}-\frac{\dot{A} \dot{C}}{A C}\right) \\
& =\left(\frac{\alpha_{1}}{t}+\alpha_{2}\right)^{2}\left(\frac{k-1}{k+1}\right)^{2}
\end{aligned}
$$

The average anisotropy parameter is

$$
\begin{aligned}
\Delta & =\frac{1}{3} \sum_{i=1}^{3}\left(\frac{H_{i}-H}{H}\right)^{2} \\
& =\frac{2}{3}\left(\frac{k-1}{k+1}\right)^{2}
\end{aligned}
$$

The deceleration parameter is

$$
\begin{aligned}
q & =-\frac{a \ddot{a}}{\dot{a}^{2}} \\
& =-1+\frac{\alpha_{1}}{\left(\alpha_{1}+\alpha_{2} t\right)^{2}}
\end{aligned}
$$

From Eqs. (1) and (21), we have the energy density of modified holographic Ricci dark energy as

$$
\rho_{\lambda}=3\left(\eta_{1}\left(\frac{\alpha_{1}}{t}+\alpha_{2}\right)-\frac{\eta_{2} \alpha_{1}}{t^{2}}+\frac{2 \alpha_{1} \eta_{3}}{3 t^{2}\left(\alpha_{1}+\alpha_{2} t\right)}\right)
$$

From Eqs. (7) and (18) the EoS parameter of modified holographic Ricci dark energy can be found as

$$
\omega_{\lambda}=\frac{\frac{2 \alpha_{1}}{t^{2}}-\frac{4 k^{2}}{(k+1)^{2}}\left(\frac{\alpha_{1}}{t}+\alpha_{2}\right)^{2}+\frac{1}{\left(a_{0} t^{\alpha_{1}} e^{\alpha_{2} t}\right)^{2}}+\frac{w \varphi_{0}}{2\left(a_{0} t^{\alpha_{1}} e^{\alpha_{2} t}\right)^{6}}}{3\left(\eta_{1}\left(\frac{\alpha_{1}}{t}+\alpha_{2}\right)-\frac{\eta_{2} \alpha_{1}}{t^{2}}+\frac{2 \alpha_{1} \eta_{3}}{3 t^{2}\left(\alpha_{1}+\alpha_{2} t\right)}\right)}
$$

From Eqs. (8), (9), (18), (26) and (27), we get the skewness parameters as 


$$
\begin{gathered}
\delta_{y}=\frac{\frac{\left(3 k^{2}-4 k-3\right)}{(k+1)^{2}}\left(\frac{\alpha_{1}}{t}+\alpha_{2}\right)^{2}-\frac{2 k \alpha_{1}}{(k+1) t^{2}}}{3\left(\eta_{1}\left(\frac{\alpha_{1}}{t}+\alpha_{2}\right)-\frac{\eta_{2} \alpha_{1}}{t^{2}}+\frac{2 \alpha_{1} \eta_{3}}{3 t^{2}\left(\alpha_{1}+\alpha_{2} t\right)}\right)} \\
\delta_{z}=\frac{\frac{2(k-1) \alpha_{1}}{(k+1) t^{2}}-\frac{(3 k+1)(k-1)}{(k+1)^{2}}\left(\frac{\alpha_{1}}{t}+\alpha_{2}\right)^{2}}{3\left(\eta_{1}\left(\frac{\alpha_{1}}{t}+\alpha_{2}\right)-\frac{\eta_{2} \alpha_{1}}{t^{2}}+\frac{2 \alpha_{1} \eta_{3}}{3 t^{2}\left(\alpha_{1}+\alpha_{2} t\right)}\right)}
\end{gathered}
$$

From Eqs. (10), (18) and (26), we have the energy density of matter as

$$
\rho_{M}=\frac{2\left(k^{2}+4 k+1\right)}{(k+1)^{2}}\left(\frac{\alpha_{1}}{t}+\alpha_{2}\right)^{2}-\frac{3}{\left(a_{0} t^{\alpha_{1}} e^{\alpha_{2} t}\right)^{2}}+\frac{w \varphi_{0}}{2\left(a_{0} t^{\alpha_{1}} e^{\alpha_{2} t}\right)^{6}}-3\left(\eta_{1}\left(\frac{\alpha_{1}}{t}+\alpha_{2}\right)-\frac{\eta_{2} \alpha_{1}}{t^{2}}+\frac{2 \alpha_{1} \eta_{3}}{3 t^{2}\left(\alpha_{1}+\alpha_{2} t\right)}\right)
$$

Now we shall discuss the physical significance of the above parameters. It is observed that the spatial volume $\mathrm{V}$ at $t=0$ vanish. The average Hubble parameter $H$, expansion scalar $\theta$, and the shear scalar $\sigma^{2}$ all diverge at $t=0$. Hence the model has a point type singularity. As time increase $H, \theta \quad \sigma^{2}$ and $\varphi$ decrease. Fig-1 shows the behavior of the deceleration parameter versus cosmic time in this model. It can be observed that the model is varying from early decelerated $(q>0)$ phase to present accelerating $(q<0)$ phase. It can, also, be observed that at $t \rightarrow \infty, q=-1$ and $\frac{d H}{d t}=0$ which implies the greatest value of Hubble parameter $H$ and fastest rate of expansion of the universe. Also, $\rho_{\lambda}, \rho_{M}, \delta_{y}$ and $\delta_{z}$ diverse at $t=0$ and decrease as the universe expands. The behavior of EoS parameter of this model is depicted in Fig. 2. It is observed that the EoS parameter is always varying in quintessence region $\left(\omega_{\lambda}>-1\right)$ in this model.

$$
\text { It can be seen that } \frac{\sigma^{2}}{\theta^{2}} \neq 0 \text { and the average anisotropy parameter } \Delta \neq 0 \text { except at }
$$
$k=1$. This implies that our model is anisotropic at all times except when $k=1$. It may be mentioned here that in the case of Sarkar [26] the universe approaches isotropy at late 
times and in the case of Sarkar [27] it remains anisotropic throughout its evolution. Also when $k=1$, we have $\sigma^{2}=0$. Hence our model in this case becomes shear free.

Model-2: We assume the average scale factor suggested by Mishra et al. [28, 29],

$$
a(t)=[\sinh (\alpha t)]^{\frac{1}{n}}
$$

This yields a time dependent deceleration parameter. Also, recently, Reddy et al. [30] have discussed Bianchi type-III dark energy model in $f(R, T)$ gravity with a variable deceleration parameter.

Using equations (15), (16) and (31) we obtain the scale factors as

$$
\begin{aligned}
& A=[\sinh (\alpha t)]^{\frac{1}{n}} \\
& B=[\sinh (\alpha t)]^{\frac{2 k}{n(k+1)}} \\
& C=[\sinh (\alpha t)]^{\frac{2}{n(k+1)}}
\end{aligned}
$$

From Eqs. (12) and (32), we have the scalar field as

$$
\varphi^{\frac{n+2}{2}}=\frac{n(n+2) \varphi_{1}}{2(n-3)} \frac{[\sinh (\alpha t)]^{\frac{n-3}{3}}}{\alpha(\cosh \alpha t)}
$$

Using Eq. (32) in Eq. (2) we obtain the following model:

$$
d s^{2}=d t^{2}-[\sinh (\alpha t)]^{\frac{2}{n}} d x^{2}-[\sinh (\alpha t)]^{\frac{4 k}{n(k+1)}} e^{-2 x} d y^{2}-[\sinh (\alpha t)]^{\frac{4}{n(k+1)}} e^{-2 x} d z^{2}
$$

\section{Physical discussion of the model}

Equation (33) describes the modified holographic Ricci dark energy model in Bianchi type-V space time in the frame work of Saez-Ballester scalar-tensor theory of gravitation with the following physical and kinematical parameters which are very important for physical discussion of the model. 
Spatial volume is

$$
V=[\sinh (\alpha t)]^{\frac{3}{n}}
$$

The average Hubble's parameter is

$$
H=\frac{\dot{a}}{a}=\frac{\alpha}{n} \operatorname{coth} \alpha t
$$

The scalar expansion is

$$
\theta=3 H=\frac{3 \alpha}{n} \operatorname{coth} \alpha t
$$

The shear scalar is

$$
\sigma^{2}=\left(\frac{k-1}{k+1}\right)^{2}\left(\frac{\alpha}{n} \operatorname{coth} \alpha t\right)^{2}
$$

The average anisotropy parameter is

$$
\Delta=\frac{2}{3}\left(\frac{k-1}{k+1}\right)^{2}
$$

The deceleration parameter is

$$
q=-1+n(\sec h \alpha t)^{2}
$$

From Eqs. (1) and (35), we have the energy density of modified holographic Ricci dark energy as

$$
\rho_{\lambda}=3 \alpha^{2}\left(\frac{\eta_{1}}{n^{2}} \operatorname{coth}^{2} \alpha t+\left(2 \eta_{3}-\frac{\eta_{2}}{n}\right) \operatorname{csch}^{2} \alpha t\right)
$$

From Eqs. (7) and (32) the EoS parameter of modified holographic Ricci dark energy can be found as

$$
\omega_{\lambda}=\frac{\frac{2 \alpha^{2}}{n} \operatorname{csch} \alpha t-\frac{4 \alpha^{2} k^{2}}{n^{2}(k+1)^{2}} \operatorname{coth}^{2} \alpha t+\frac{1}{(\sinh \alpha t)^{\frac{2}{n}}}+\frac{w \varphi_{0}}{2(\sinh \alpha t)^{\frac{6}{n}}}}{3 \alpha^{2}\left(\frac{\eta_{1}}{n^{2}} \operatorname{coth}^{2} \alpha t+\left(2 \eta_{3}-\frac{\eta_{2}}{n}\right) \operatorname{csch} h^{2} \alpha t\right)}
$$


From Eqs. (8), (9), (32), (40) and (41), we get the skewness parameters as

$$
\begin{gathered}
\delta_{y}=\frac{\frac{\alpha^{2}\left(3 k^{2}-4 k-3\right)}{n^{2}(k+1)^{2}} \operatorname{coth}^{2} \alpha t-\frac{2 k \alpha^{2}}{n(k+1)} \csc h^{2} \alpha t}{3 \alpha^{2}\left(\frac{\eta_{1}}{n^{2}} \operatorname{coth}^{2} \alpha t+\left(2 \eta_{3}-\frac{\eta_{2}}{n}\right) \csc h^{2} \alpha t\right)} \\
\delta_{z}=\frac{\frac{(k-1) \alpha^{2}}{n(k+1)} \csc h^{2} \alpha t-\frac{(3 k+1)(k-1) \alpha^{2}}{n^{2}(k+1)^{2}} \operatorname{coth}^{2} \alpha t}{3 \alpha^{2}\left(\frac{\eta_{1}}{n^{2}} \operatorname{coth}^{2} \alpha t+\left(2 \eta_{3}-\frac{\eta_{2}}{n}\right) \csc h^{2} \alpha t\right)}
\end{gathered}
$$

From Eqs. (10), (32) and (40), we have the energy density of matter as

$$
\rho_{M}=\frac{2 \alpha^{2}\left(k^{2}+4 k+1\right)}{n^{2}(k+1)^{2}} \operatorname{coth}^{2} \alpha t-\frac{3}{(\sinh \alpha t)^{\frac{2}{n}}}+\frac{w \varphi_{0}}{2(\sinh \alpha t)^{\frac{6}{n}}}-3 \alpha^{2}\left(\frac{\eta_{1}}{n^{2}} \operatorname{coth}^{2} \alpha t+\left(2 \eta_{3}-\frac{\eta_{2}}{n}\right) \csc h^{2} \alpha t\right)
$$

It can be observed from the above results the behavior of the physical and kinematical parameters is quite similar to the behavior of the parameters of the Model-1. Also, the behavior of deceleration parameter and EoS parameter for this model is depicted in Figs. 1 and 2. This model, also, is always varying in quintessence region $\left(\omega_{\lambda}>-1\right)$ and attains a constant value in that region.

Model-3: The linearly varying deceleration parameter ([16]) after taking suitable choice of integrating constants, leads to

$$
a(t)=e^{\frac{2}{m} \arctan h\left(\frac{l t}{m}-1\right)}
$$

where $l>0, m \geq 0$ for simplicity. The reason for considering the solution for $l>0$ and $m>0$ is not only for simplicity but also for compatibility with the observed universe. Here $l>0$ means we are dealing with increasing acceleration $(\dot{q}=-l<0)$. 
Recently, Reddy et al. [15] have investigated cosmological models with a linearly varying deceleration parameter in alternative theories of gravitation.

Using equations (15), (16) and (45) we obtain the scale factors as

$$
\begin{aligned}
& A=e^{\frac{2}{m} \arctan h\left(\frac{l t}{m}-1\right)} \\
& B=e^{\frac{4 k}{m(k+1)} \arctan h\left(\frac{l t}{m}-1\right)} \\
& C=e^{\frac{4}{m(k+1)} \arctan h\left(\frac{l t}{m}-1\right)}
\end{aligned}
$$

From Eqs. (12) and (46), we have the scalar field as

$$
\varphi^{\frac{n+2}{2}}=\frac{\varphi_{2}(n+2)}{12 l}\left(l^{2} t^{2}-2 \operatorname{lm} t\right) e^{\frac{-6}{m} \arctan h\left(\frac{l t}{m}-1\right)}+\psi_{2}
$$

Using Eq. (46) in Eq. (2) we obtain the following model:

$d s^{2}=d t^{2}-e^{\frac{8}{m} \arctan h\left(\frac{l t}{m}-1\right)} d x^{2}-e^{\frac{8 k}{m(k+1)} \arctan h\left(\frac{l t}{m}-1\right)} e^{-2 x} d y^{2}-e^{\frac{8}{m(k+1)} \arctan h\left(\frac{l t}{m}-1\right)} e^{-2 x} d z^{2}$

\section{Physical discussion of the model}

Equation (47) describes the modified holographic Ricci dark energy model in Bianchi type-V space time in the frame work of Saez-Ballester scalar-tensor theory of gravitation with the following physical and kinematical parameters which are very important for physical discussion of the model.

Spatial volume is

$$
V=e^{\frac{6}{m} \arctan h\left(\frac{l t}{m}-1\right)}
$$

The average Hubble parameter is

$$
H=\frac{\dot{a}}{a}=\frac{2 m}{l t(2 m-l t)}
$$

The scalar expansion is 


$$
\theta=3 H=\frac{6 m}{l t(2 m-l t)}
$$

The shear scalar is

$$
\sigma^{2}=\frac{4(k-1)^{2} m^{2}}{l^{2}(k+1)^{2} t^{2}(2 m-l t)^{2}}
$$

The average anisotropy parameter is

$$
\Delta=\frac{2}{3}\left(\frac{k-1}{k+1}\right)^{2}
$$

The deceleration parameter is

$$
q=-l t+m-1
$$

From Eqs. (1) and (49), we have the energy density of modified holographic Ricci dark energy as

$$
\rho_{\lambda}=6\left(\frac{2 \eta_{1}-2 \eta_{2}(l t-m)-\eta_{3}\left(m^{2}+(l t-m)^{2}\right)}{t^{2}(2 m-l t)^{2}}\right)
$$

From Eqs. (7) and (46) the EoS parameter of modified holographic Ricci dark energy can be found as

$$
\omega_{\lambda}=\frac{\frac{8(m-l t)(k+1)^{2}-16(k+1)-16 k^{2}}{t^{2}(k+1)^{2}(2 m-l t)^{2}}+\exp \left(\frac{-4}{m} \arctan h\left(\frac{l t}{m}-1\right)\right)+\frac{w \varphi_{0}}{2} \exp \left(\frac{-12}{m} \arctan h\left(\frac{l t}{m}-1\right)\right)}{6\left(\frac{2 \eta_{1}-2 \eta_{2}(l t-m)-\eta_{3}\left(m^{2}+(l t-m)^{2}\right)}{t^{2}(2 m-l t)^{2}}\right)}(55)
$$

From Eqs. (8), (9), (46), (54) and (55), we get the skewness parameters as

$$
\begin{aligned}
& \delta_{y}=\frac{8(l t-m)+12(k-1)}{(k+1)\left(2 \eta_{1}-2 \eta_{2}(l t-m)-\eta_{3}\left(m^{2}+(l t-m)^{2}\right)\right)} \\
& \delta_{z}=\frac{(4(m-l t)-12)(k-1)}{(k+1)\left(2 \eta_{1}-2 \eta_{2}(l t-m)-\eta_{3}\left(m^{2}+(l t-m)^{2}\right)\right)}
\end{aligned}
$$

From Eqs. (10), (46) and (54), we have the energy density of matter as 


$$
\begin{aligned}
\rho_{M}=\frac{8\left(k^{2}+4 k+1\right)}{t^{2}(k+1)^{2}(2 m-l t)^{2}}-3 \exp \left(\frac{-4}{m} \arctan h\left(\frac{l t}{m}-1\right)\right) & +\frac{w \varphi_{0}}{2} \exp \left(\frac{-12}{m} \arctan h\left(\frac{l t}{m}-1\right)\right) \\
& -6\left(\frac{2 \eta_{1}-2 \eta_{2}(l t-m)-\eta_{3}\left(m^{2}+(l t-m)^{2}\right)}{t^{2}(2 m-l t)^{2}}\right)
\end{aligned}
$$

The spatial volume exhibits an exponential increase with cosmic time which implies inflation. The parameters $H, \theta \sigma^{2}, \rho_{M}, \rho_{\lambda}$ and $\varphi$ diverse at initial epoch and they all decrease as time increases. Also at $k=1, \Delta=0$ and $\sigma^{2}=0$ which imply that this model is isotropic and shear free for this value of $k$. Also the skewness parameters $\delta_{y}$ and $\delta_{z}$ are constant at $t=0$ and they vanish as $t \rightarrow \infty$. The deceleration parameter and the EoS parameter of this model are plotted in Figs. 1 and 2. It can be observed this model is also evolving from early decelerated $(q>0)$ phase to present accelerating $(q<0)$ phase. It can be seen that the model in this case always, remains in the phantom region $\left(\omega_{\lambda}<-1\right)$.

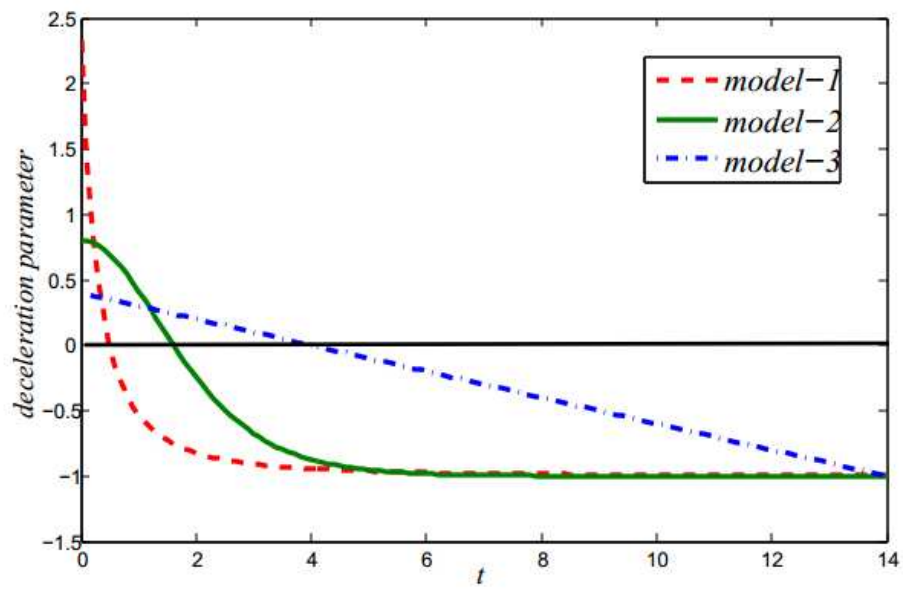

Fig.1:Plot of deceleration parameter versus time. 

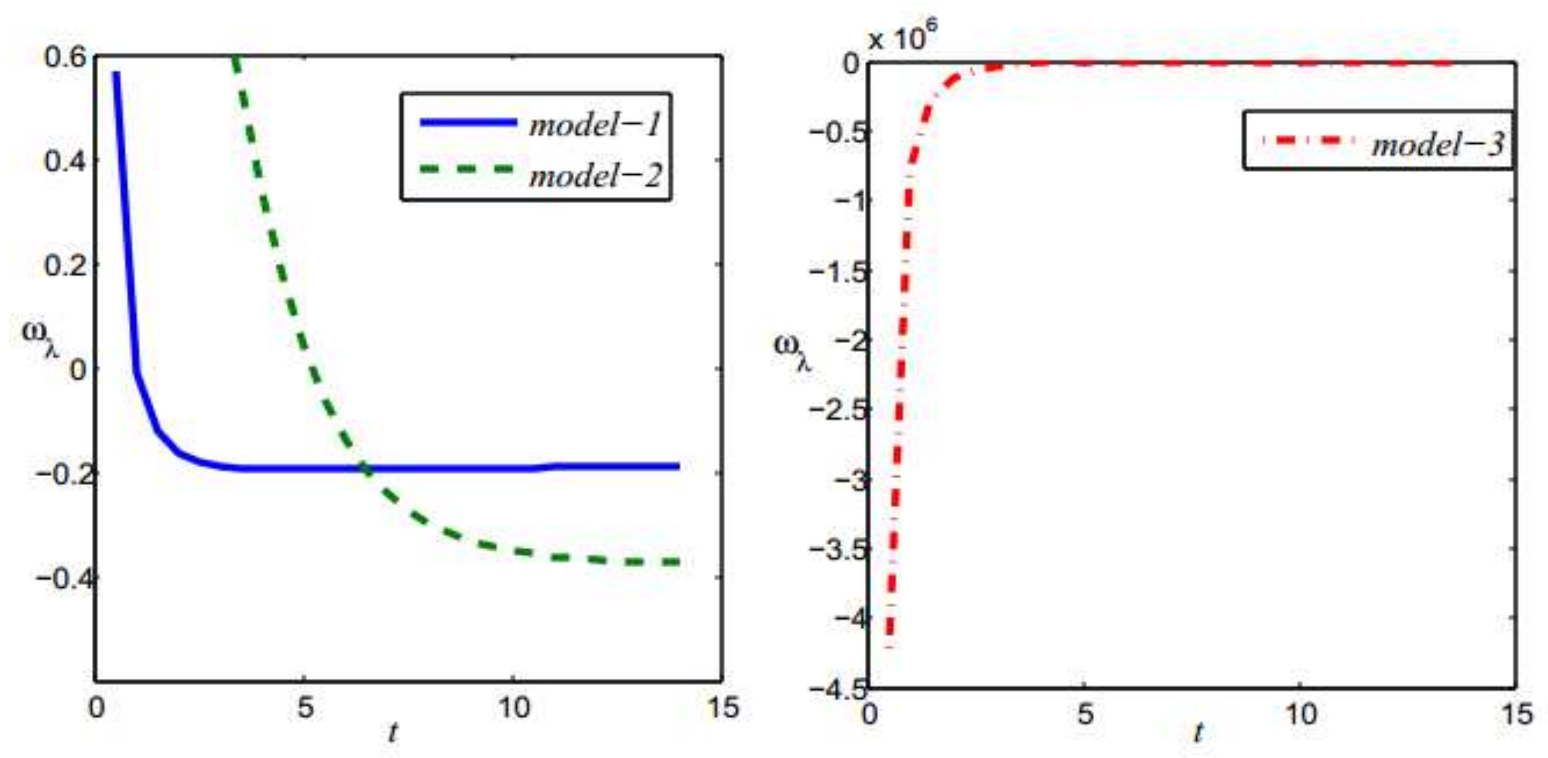

Fig.2: Plots of EoS parameter versus time.

\section{Conclusions}

In this paper, we have investigated spatially homogeneous and anisotropic modified holographic Ricci dark energy Bianchi type- $\mathrm{V}$ cosmological models in Saez-Ballester [21] scalar-tensor theory of gravitation.We have obtained three different models using hybrid expansion law of average scale factor [23], varying deceleration parameters $[28,16]$. The entire above three models exhibit a smoothtransition of the universe from the decelerated phase to the accelerating phase. It can be seen that at the initial epoch the spatial volume of the models vanish showing that there exists a point type singularity at $t=0$. We have found all the physical and kinematical parameters corresponding to each modeland it is observed that they all diverse at the initial epoch. It can be seen that in all the models spatial volume becomes infinite as $\quad t \rightarrow \infty$ while Hubble's parameter $H$ is constant for the model (19). Hence, in this particular case, the universe expands forever with constant rate. We have also studied the behavior of EoS parameter in 
the models. It is interesting to observe that the models with hybrid expansion law and with varying deceleration parameter always vary in the quintessence region while the model with linearly varying deceleration parameter varies in phantom region. This may be due to the fact that we are considering scalar field models. It may also be noted that the Saez-Ballester scalar field $\varphi$ goes to infinity as $t \rightarrow \infty$ whereas it becomes zero when $t=0$.

Acknowledgements: The author is very much thankful to the reviewer for giving constructive comments which have helped to improve the presentation of the paper.

\section{References}

[1] S. Perlmutter, et al.: Astrophys. J. 517, 565(1999).

[2] A. Riess, et al.: Astron. J., 116, 1009 (1998).

[3] G. Hoof't: gr-qc/9310026 (1995)

[4] A. Cohen, et al.: Phys. Rev. Lett. 82, 4971 (1999).

[5] S.D.H. Hsu.: Phy. Lett., 594, 13 (2014).

[6] C. Gao, et al.: Phys. Rev. D, 79, 043511 (2009)

[7] L.N. Granda, A. Oliveros, Phys.Lett. B, 669, 275 (2008).

[8] S. Chen, J. Jing, Phys. Lett. B, 679, 144 (2009)

[9] S. Sarkar, Astrophys. Space Sci., 352, 245 (2014).

[10] K.S. Adhav, et al., Astrophys. Space Sci. 359, 24 (2015).

[11] M. Kiran, et al., Astrophys. Space Sci., 354, 2099 (2014).

[12] M. Kiran, et al.: Astrophys. Space Sci., 356, 407 (2015).

[13] S. Umadevi, G. Ramesh: Astrophys. Space Sci., 359, 51 (2015).

[14] M. Kiran, et al.: Astrophys. Space Sci., 360, 54 (2015).

[15] D.R.K. Reddy, et al.: Prespacetime J., 6, 1171(2015).

[16] O. Akarsu, T. Dereli, Int. J. Theor. Phys. 51, 612 (2012)

[17] K. Das, T. Sultana: Astrophys. Space Sci.360, 4 (2015)

[18] K. Das, T. Sultana: Astrophys. Space Sci..361, 53 (2016).

[19] M. Azizur Rahman, M. Ansari, Astrophys. Space Sci. 354, 617 (2014)

[20] M. Azizur Rahman, M. Ansari, Astrophys. Space Sci. 354, 675 (2014).

[21] D. Saez, V.J. Ballester, Phys. Lett. A., 113, 467 (1986).

[22] C. B. Collins, et al.: Gen. Relativ. Gravit. 12, 805 (1983).

[23] O. Akarsu, et al., JCAP, 01, 022 (2014) 
Page 17 of 17

Canadian Journal of Physics

[24] Shri Ram, S. Chandel: Astrophys. Space Sci. 355, 195 (2015).

[25] M.V. Santhi, et al.: Astrophys. Space Sci., 361, 142 (2016).

[26] S. Sarkar: Astrophys. Space Sci. 349, 985 (2014)

[27] S. Sarkar: Astrophys. Space Sci. 350, 821 (2014)

[28] R.K. Mishra, et al.: Int. J. Theor. Phys., 52, 2546 (2013).

[29] R.K. Mishra, et al.: Int. J. Theor. Phys.,doi 10.1007/s10773-015-2766-0 ( 2015)

[30] D.R.K.Reddy et al.: arxiv: 1601. 02648v1 (Phys. Gen. ph) (2016).

https://mc06.manuscriptcentral.com/cjp-pubs 